\title{
An abstract proximal point algorithm
}

\author{
Laurenţiu Leuştean ${ }^{a, b, c}$, Adriana Nicolae ${ }^{d, e}$ and Andrei Sipoş ${ }^{f, c}$ \\ ${ }^{a}$ The Research Institute of the University of Bucharest (ICUB), University of Bucharest, \\ M. Kogălniceanu 36-46, 050107, Bucharest, Romania \\ ${ }^{b}$ Faculty of Mathematics and Computer Science, University of Bucharest, \\ Academiei 14, 010014, Bucharest, Romania \\ ${ }^{c}$ Simion Stoilow Institute of Mathematics of the Romanian Academy, \\ Calea Griviţei 21, 010702, Bucharest, Romania \\ ${ }^{d}$ Department of Mathematical Analysis - IMUS, University of Seville, \\ C/ Tarfia s/n, 41012 Sevilla, Spain \\ ${ }^{e}$ Department of Mathematics, Babeş-Bolyai University, \\ Kogălniceanu 1, 400084 Cluj-Napoca, Romania \\ ${ }^{f}$ Department of Mathematics, Technische Universität Darmstadt, \\ Schlossgartenstrasse 7, 64289 Darmstadt, Germany
}

E-mails: laurentiu.leustean@unibuc.ro, anicolae@math.ubbcluj.ro, sipos@mathematik.tu-darmstadt.de

\begin{abstract}
The proximal point algorithm is a widely used tool for solving a variety of convex optimization problems such as finding zeros of maximally monotone operators, fixed points of nonexpansive mappings, as well as minimizing convex functions. The algorithm works by applying successively so-called "resolvent" mappings associated to the original object that one aims to optimize. In this paper we abstract from the corresponding resolvents employed in these problems the natural notion of jointly firmly nonexpansive families of mappings. This leads to a streamlined method of proving weak convergence of this class of algorithms in the context of complete CAT(0) spaces (and hence also in Hilbert spaces). In addition, we consider the notion of uniform firm nonexpansivity in order to similarly provide a unified presentation of a case where the algorithm converges strongly. Methods which stem from proof mining, an applied subfield of logic, yield in this situation computable and low-complexity rates of convergence.
\end{abstract}

Keywords: Convex optimization; Proximal point algorithm; CAT(0) spaces; Jointly firmly nonexpansive families; Uniformly firmly nonexpansive mappings; Proof mining; Rates of convergence.

Mathematics Subject Classification 2010: 90C25, 46N10, 47J25, 47H09, 03F10

\section{Introduction}

The first instance of what came later to be known as the proximal point algorithm can be found in a short communication from 1970 of Martinet [44]. He considered (among others) the issue of solving the minimization problem

$$
\operatorname{argmin}_{x \in C} f(x),
$$


where $C$ is a closed convex subset of a Hilbert space $H$ and $f$ is a (real-valued) lower semicontinuous convex function defined on $C$, that further has the property that for all $a \in \mathbb{R}$, the set

$$
\{x \in C \mid f(x) \leq a\}
$$

is bounded. One then starts from an arbitrary point $x_{0} \in C$ and afterwards iteratively builds a sequence $\left(x_{n}\right)$ by the implicit (though uniquely determining) recurrence relation

$$
f\left(x_{n+1}\right)=\min _{y \in C}\left(f(y)+\left\|x_{n}-y\right\|^{2}\right) .
$$

Martinet's Théorème 3 then asserts that any weak cluster point of this sequence is a solution to the given minimization problem.

In 1976, Rockafellar [47] took up the more general problem of finding a zero of a maximally monotone multi-valued operator $A: H \rightarrow 2^{H}$, i.e. a point $x$ such that $0 \in A(x)$ (single-valued monotone operators had already been considered by Martinet). This contains the previous case since the subdifferential $\partial f$ of a function $f$ having the properties considered above is a maximally monotone operator whose zeros coincide with the minimizers of $f$. The method used in this case in order to approach the desired solution was called the "proximal point algorithm" and generates starting from a point $x_{0} \in C$ a sequence using another implicit recurrence, namely

$$
x_{n} \in\left(i d_{H}+\gamma_{n} A\right)\left(x_{n+1}\right),
$$

where $\left(\gamma_{n}\right)$ is a sequence of positive real numbers. When $A=\partial f$, the relation reduces to the previous one if $\left(\gamma_{n}\right)$ is the sequence constantly equal to $1 / 2$. Theorem 1 of [47] shows that if

$$
\inf _{n \in \mathbb{N}} \gamma_{n}>0,
$$

then $\left(x_{n}\right)$ weakly converges to a zero of $A$. Strong convergence is proved under some additional uniformity assumptions (such as $A^{-1}$ being Lipschitz continuous at 0 ), but it does not generally hold, as Güler [21, Corollary 5.1] later put forward a counterexample in this sense. Two years after Rockafellar's paper, Brézis and Lions [12] studied more general conditions one could impose on $\left(\gamma_{n}\right)$ that still yield weak convergence of the sequence $\left(x_{n}\right)$, there regarded as the "infinite product" of the resolvent operators

$$
J_{\gamma_{n} A}:=\left(i d_{H}+\gamma_{n} A\right)^{-1} .
$$

Those conditions continue to be the state of the art - e.g. for an arbitrary maximally monotone operator one may assume ([12, Proposition 8])

$$
\sum_{n=0}^{\infty} \gamma_{n}^{2}=\infty
$$

The proximal point algorithm has grown to become a versatile tool of convex optimization, being used, in addition to the applications already expounded upon, to solve a plethora of problems such as variational inequalities, minimax or equilibrium problems (some of these may already be found in the papers cited above). The book of Bauschke and Combettes [9] may serve as an introduction to the field in the context of Hilbert spaces.

Outside Hilbert spaces, a natural generalization of the resolvent operator was given in the 1990s by Jost [25] and Mayer [45] in the context of complete CAT(0) spaces, which can be regarded as the proper nonlinear analogue of Hilbert spaces. Using this definition and an appropriate notion of weak convergence introduced by Lim [43], called $\Delta$-convergence, Bačák [3] extended in 2013 Théorème 9 of Brézis and Lions [12] in this context. More precisely, he proved the $\Delta$-convergence of the sequence generated by the proximal point algorithm when $f$ is a proper, convex and lower semicontinuous function that attains its minimum.

A separate strand of development came from fixed point theory. In the 1960s, Browder [15] and Halpern [22] studied the existence and computation of fixed points of nonexpansive mappings 
$T: C \rightarrow C$ (where $C$ is a closed convex bounded subset of a Hilbert space). They considered the notion of a resolvent of order $\gamma$ of $T$ - that is, a mapping satisfying, for all $x \in C$,

$$
R_{\gamma} x=\frac{1}{1+\gamma} x+\frac{\gamma}{1+\gamma} T R_{\gamma} x .
$$

In the above, we reparametrized their construction in order to obtain a better fit with the objects considered here. Their main result states that by letting $\gamma \rightarrow \infty, R_{\gamma} x$ tends to the fixed point of $T$ which is the closest to $x$. Halpern's particularly simple argument was later generalized to the Hilbert ball in [20] and to complete CAT(0) spaces in [27]. A proof in the latter setting that starts from minimal boundedness assumptions may be found in a 2014 paper of Bačák and Reich [6]. Note that [6] also contains a variant of the proximal point algorithm which constructs, by iterating the resolvents of $T$, a sequence that $\Delta$-converges to a fixed point of $T$.

As one may notice, every iterative sequence that was considered above under the name of "proximal point algorithm" follows a pattern: we have a mathematical object that we seek to optimize in some way, we construct associated "resolvent" operators, we take an initial arbitrary point $x$ and finally we iterate those operators starting from $x$. One may ask whether there are some very general hypotheses which yield the convergence of the resulting sequence without explicitly considering the particular details of the optimization problem at hand. Our first main goal is to answer this question in the affirmative in the framework of CAT(0) spaces (and therefore also for Hilbert spaces) by deriving some conditions related to firm nonexpansivity that are satisfied by all the above types of families of resolvents. We give these conditions in weaker and stronger forms, and show that while the strongest one is generally satisfied, the weakest one suffices to obtain appropriate convergence results. We should mention here that it was known for a long time that individual resolvents are in particular firmly nonexpansive, and some abstract results in the same spirit were previously obtained by Ariza-Ruiz, the first author and López-Acedo in [1]. The present paper may be regarded as a natural continuation of the study initiated there (see also [2]).

Section 2 introduces general notions and properties regarding geodesic metric spaces and mappings that are used in the sequel. Section 3 starts with some very general hypotheses for a sequence generated by a family of firmly nonexpansive mappings that yield its weak or $\Delta$-convergence. In the process, we derive some lemmas that characterize various asymptotic aspects of the proximal point algorithm. Then we define two conditions that one may impose on a family $\left(T_{n}\right)$ with respect to a sequence $\left(\gamma_{n}\right)$. These conditions generalize the property of a mapping being firmly nonexpansive to a relation between two possibly different mappings which is then applied to each possible pair from the countable family. We claim that these definitions capture the residual property used in convergence proofs that corresponds to the way a family of resolvents $\left(J_{\gamma_{n}}\right)$ behaves with respect to the sequence of step-sizes $\left(\gamma_{n}\right)$. We consider then "jointly firmly nonexpansive families" and a somewhat weaker notion, "jointly $\left(P_{2}\right)$ families" from which the general conditions can be obtained. In particular, the families of mappings involved in the problems discussed before (i.e. minimization of convex functions, finding fixed points of nonexpansive mappings and finding zeros of maximally monotone operators) satisfy these conditions.

The second main goal of this paper is to find quantitative variants of some convergence results for the proximal point algorithm. This falls within the purview of proof mining, an applied subfield of logic. Proof mining primarily concerns itself with the application of tools from proof theory to obtain computational content for theorems in ordinary mathematics with proofs that are not necessarily fully constructive. The project was first suggested in the 1950s by Kreisel under the name of "unwinding of proofs", but it gained considerable momentum after its extensive development in the 1990s and 2000s by Kohlenbach and his collaborators, culminating with the publication of general logical metatheorems, developed by Kohlenbach [30] and by Gerhardy and Kohlenbach [19], that tell us when a proof of a theorem proven in classical logic may be analyzed in order to obtain ("extract") its hidden quantitative information. A comprehensive reference for the major developments of the field up to 2008 is the monograph of Kohlenbach [31], while surveys of recent results are [32, 33]. So far, proof mining has been successfully applied to obtain quantitative versions of celebrated results in various areas of mathematics such as approximation 
theory, nonlinear analysis, metric fixed point theory, ergodic theory, or topological dynamics. Recently, its methods have begun to be applied to convex optimization, for more details see $[5,34,35,36,38,41,42]$.

Let us discuss the sort of quantitative results that we obtain. If $\left(x_{n}\right)$ is a sequence in a metric space $X$ and $x \in X$, then $\lim _{n \rightarrow \infty} x_{n}=x$ if and only if

$$
\forall k \in \mathbb{N} \exists N \in \mathbb{N} \forall n \geq N\left(d\left(x_{n}, x\right) \leq \frac{1}{k+1}\right) .
$$

A quantitative version of the above would be a rate of convergence for the sequence: a formula showing how to compute the $N$ in terms of the $k$. However, very simple real-valued sequences have been shown by methods of mathematical logic to lack a computable rate of convergence. We recall, though, from the discussion above, that strong convergence of the proximal point algorithm could only be obtained under some extra uniformity assumptions. Fortunately, some of these conditions yield the uniqueness of the needed optimizing point (minimizer, fixed point or zero). This uniqueness was shown by the work of Kohlenbach [29], Kohlenbach and Oliva [37, Section 4.1] and Briseid [14] to guarantee the extraction of a rate of convergence, relative to some other piece of quantitative information. In Section 4, therefore, we define a general notion of uniformity applicable to our families of mappings (extending the similar notion given in [8] in the context of Hilbert spaces). One then shows that the concrete algorithms have corresponding "uniform" cases that are subsumed into this definition, e.g. finding zeros of uniformly monotone mappings or minimizing uniformly convex functions.

Section 5 then shows that this definition suffices: a quantitative variant of the asymptotic lemmas from Section 3 fits in as the relevant piece of information that is then used, as per the above discussion, to obtain a highly uniform rate of convergence for this special case of the proximal point algorithm. As a byproduct, we obtain an alternate proof for the classical qualitative results of strong convergence.

Proximal methods are not limited to the classical problems of convex optimization. Therefore, a question that arises is to what extent a natural and abstract approach of the type provided here could be employed to capture other such variants, which are used, for example, in global (nonconvex) optimization $[26,23]$, where the necessity of the existence of iterates requires one to assume weak forms of monotonicity. Another direction consists in considering multi-valued resolvent-type operators instead of single-valued ones. In this case the algorithm becomes nondeterministic (i.e. given a current iterate, the following one is not uniquely determined). Such a development would allow one to cover e.g. vector-valued optimization problems $[11,16]$.

\section{Preliminaries}

We start by briefly recalling some notions and properties about geodesic spaces needed in the sequel. More details on geodesic spaces can be found, for example, in $[46,13,4]$. Let $(X, d)$ be a metric space. A geodesic in $X$ is a mapping $\gamma:[a, b] \rightarrow X$ (where $a, b \in \mathbb{R}$ ) such that for all $s, t \in[a, b]$,

$$
d(\gamma(s), \gamma(t))=|s-t| .
$$

We say that $X$ is a geodesic space if for all $x, y \in X$, there is a geodesic $\gamma:[a, b] \rightarrow X$ satisfying $\gamma(a)=x$ and $\gamma(b)=y$.

A geodesic space $(X, d)$ is called a $C A T(0)$ space if for all $z \in X$, all geodesics $\gamma:[a, b] \rightarrow X$ and all $t \in[0,1]$ we have that

$$
d^{2}(z, \gamma((1-t) a+t b)) \leq(1-t) d^{2}(z, \gamma(a))+t d^{2}(z, \gamma(b))-t(1-t) d^{2}(\gamma(a), \gamma(b)) .
$$

It easily follows that every CAT(0) space is uniquely geodesic - that is, for any $x, y$ in such a space $X$ there is a unique geodesic $\gamma:[0, d(x, y)] \rightarrow X$ such that $\gamma(0)=x$ and $\gamma(d(x, y))=y-$ and in 
this framework we shall denote, for any $t \in[0,1]$, the point $\gamma(t d(x, y))$ by $(1-t) x+t y$. Note that every CAT(0) space $X$ is Busemann convex-i.e., for any $x, y, u, v \in X$ and $t \in[0,1]$,

$$
d((1-t) x+t y,(1-t) u+t v) \leq(1-t) d(x, u)+t d(y, v) .
$$

We will also make use of the quasi-linearization function $\langle\cdot, \cdot\rangle: X^{2} \times X^{2} \rightarrow \mathbb{R}$ introduced by Berg and Nikolaev in [10], which is defined, for any $x, y, u, v \in X$, by the following (where an ordered pair of points $\left(w, w^{\prime}\right) \in X^{2}$ is denoted by $\left.\overrightarrow{w w^{\prime}}\right)$ :

$$
\langle\overrightarrow{x y}, \overrightarrow{u v}\rangle:=\frac{1}{2}\left(d^{2}(x, v)+d^{2}(y, u)-d^{2}(x, u)-d^{2}(y, v)\right) .
$$

Proposition 2.1 ([10, Proposition 14]). In any metric space $(X, d)$, the mapping $\langle\cdot, \cdot\rangle$ is the unique one that satisfies, for any $x, y, u, v, w \in X$, the following properties:

(i) $\langle\overrightarrow{x y}, \overrightarrow{x y}\rangle=d^{2}(x, y)$;

(ii) $\langle\overrightarrow{x y}, \overrightarrow{u v}\rangle=\langle\overrightarrow{u v}, \overrightarrow{x y}\rangle$;

(iii) $\langle\overrightarrow{y x}, \overrightarrow{u v}\rangle=-\langle\vec{x}, \overrightarrow{u v}\rangle$;

(iv) $\langle\overrightarrow{x y}, \overrightarrow{u v}\rangle+\langle\overrightarrow{x y}, \overrightarrow{v w}\rangle=\langle\overrightarrow{x y}, \overrightarrow{u w}\rangle$.

In particular, if $X$ is a real Hilbert space with inner product $\langle\cdot, \cdot\rangle$, then

$$
\langle\overrightarrow{x y}, \overrightarrow{u v}\rangle=\langle x-y, u-v\rangle=\langle y-x, v-u\rangle,
$$

for all $x, y, u, v \in X$. This justifies the notation.

The main result of [10], Theorem 1, gives a characterization of CAT(0) spaces in terms of the "Cauchy-Schwarz" inequality for $\langle\cdot, \cdot\rangle$. More precisely, a geodesic space $(X, d)$ is $\operatorname{CAT}(0)$ if and only if

$$
\langle\overrightarrow{x y}, \overrightarrow{u v}\rangle \leq d(x, y) d(u, v)
$$

for all $x, y, u, v \in X$. Furthermore, by [10, Theorem 6], a related condition for a geodesic space $(X, d)$ to be $\operatorname{CAT}(0)$ is the following inequality

$$
d^{2}(x, v)+d^{2}(y, u) \leq d^{2}(x, u)+d^{2}(y, v)+d^{2}(x, y)+d^{2}(u, v),
$$

which is to be satisfied for all $x, y, u, v \in X$.

For the rest of the section, $(X, d)$ is a geodesic space, unless stated otherwise. If $T: X \rightarrow X$ is a mapping, we denote by Fix $(T)$ the set of its fixed points.

The following generalization of firmly nonexpansive mappings to geodesic spaces was introduced in [1].

Definition 2.2. A mapping $T: X \rightarrow X$ is called firmly nonexpansive if for any $x, y \in X$ and any $t \in[0,1]$ we have that

$$
d(T x, T y) \leq d((1-t) x+t T x,(1-t) y+t T y) .
$$

As mentioned in [2] (see also [36]), if $X$ is a CAT(0) space, every firmly nonexpansive mapping $T: X \rightarrow X$ satisfies the so-called property $\left(P_{2}\right)$. Namely,

$$
2 d^{2}(T x, T y) \leq d^{2}(x, T y)+d^{2}(y, T x)-d^{2}(x, T x)-d^{2}(y, T y),
$$

for all $x, y \in X$. In other words,

$$
d^{2}(T x, T y) \leq\langle\overrightarrow{T x T y}, \overrightarrow{x y}\rangle,
$$

for all $x, y \in X$. If $X$ is a Hilbert space, property $\left(P_{2}\right)$ is sufficient for firm nonexpansivity as (7) and (4) yield $\|T x-T y\|^{2} \leq\langle T x-T y, x-y\rangle$, which is, in turn, equivalent to Definition 2.2 (see, e.g., [9, Proposition 4.2] for a proof). Moreover, from (7) and (5) one immediately obtains the following result. 
Lemma 2.3. If $X$ is a $C A T(0)$ space and $T: X \rightarrow X$ satisfies property $\left(P_{2}\right)$, then $T$ is nonexpansive.

Let $\left(x_{n}\right)$ be a bounded sequence in $X$ and $F \subseteq X$ be nonempty. For any $y \in X$, define

$$
r\left(y,\left(x_{n}\right)\right):=\limsup _{n \rightarrow \infty} d\left(y, x_{n}\right), \quad r\left(F,\left(x_{n}\right)\right):=\inf \left\{r\left(y,\left(x_{n}\right)\right) \mid y \in F\right\} .
$$

Furthermore, $A\left(F,\left(x_{n}\right)\right):=\left\{y \in F \mid r\left(y,\left(x_{n}\right)\right)=r\left(F,\left(x_{n}\right)\right)\right\}$ and elements of $A\left(F,\left(x_{n}\right)\right)$ are called asymptotic centers of $\left(x_{n}\right)$ with respect to $F$. We shall denote $A\left(X,\left(x_{n}\right)\right)$ by $A\left(\left(x_{n}\right)\right)$ and call its elements asymptotic centers of $\left(x_{n}\right)$.

The next results will be used in the subsequent sections.

Lemma 2.4 ([40, Lemma 3.2]). Let $\left(x_{n}\right)$ be a bounded sequence in $X$ with $A\left(\left(x_{n}\right)\right)=\{c\}$ and $\left(\alpha_{n}\right),\left(\beta_{n}\right)$ be real sequences such that $\alpha_{n} \geq 0$ for all $n \in \mathbb{N}, \lim _{\sup _{n \rightarrow \infty}} \alpha_{n} \leq 1$ and $\lim \sup _{n \rightarrow \infty} \beta_{n} \leq 0$.

Assume that $y \in X$ is such that there exist $p, N \in \mathbb{N}$ satisfying, for all $n \geq N$,

$$
d\left(y, x_{n+p}\right) \leq \alpha_{n} d\left(c, x_{n}\right)+\beta_{n} .
$$

Then $y=c$.

Proposition 2.5 ([17, Proposition 7]). Every bounded sequence $\left(x_{n}\right)$ in a complete CAT(0) space $X$ has a unique asymptotic center with respect to any nonempty closed convex subset of $X$.

In order to state our main results, we need to introduce the notion of $\Delta$-convergence which was defined by Lim [43] in metric spaces. We refer to [39, 24, 18] for equivalent notions in the setting of complete CAT(0) spaces, where $\Delta$-convergence can be seen as a generalization of the weak convergence in Banach spaces (see [28]). In fact, in Hilbert spaces, $\Delta$-convergence coincides with weak convergence (see [4, Exercise 3.1]).

Definition 2.6. A bounded sequence $\left(x_{n}\right) \Delta$-converges to a point $x \in X$ if for any subsequence $\left(u_{n}\right)$ of $\left(x_{n}\right)$ we have that $A\left(\left(u_{n}\right)\right)=\{x\}$.

The notion of Fejér monotonicity will also play an important role in this work. Let $\left(x_{n}\right)$ be a sequence in $X$ and $F \subseteq X$ be nonempty.

Definition 2.7. We say that $\left(x_{n}\right)$ is Fejér monotone with respect to $F$ if for all $p \in F$ and all $n \in \mathbb{N}$, we have that

$$
d\left(x_{n+1}, p\right) \leq d\left(x_{n}, p\right)
$$

It is obvious that if $\left(x_{n}\right)$ is Fejér monotone with respect to $F$, then $\left(d\left(x_{n}, p\right)\right)$ converges for every $p \in F$ and, furthermore, $\left(x_{n}\right)$ is bounded.

Finally, we recall the following well-known result (see, for example, [7, Proposition 3.3.(iii)]), which turns out to be very useful in obtaining $\Delta$-convergence results.

Proposition 2.8. Let $X$ be a complete $C A T(0)$ space and $\left(x_{n}\right)$ be Fejér monotone with respect to $F$. Assume that the asymptotic center of every subsequence of $\left(x_{n}\right)$ is in $F$. Then $\left(x_{n}\right) \Delta$-converges to some $x \in F$.

\section{An abstract Proximal Point Algorithm}

We now begin the process of modularizing the proof(s) that guarantee the weak convergence of common instances of the proximal point algorithm. Theorem 3.5 is the first stage in this sense and provides some highly general conditions under which the iteration constructed by applying countably many mappings converges weakly. In proving it, we shall also show some fundamental properties of that iterative sequence, such as Fejér monotonicity and a form of asymptotic regularity. 
In the following, $X$ is a complete $\operatorname{CAT}(0)$ space and $\left(T_{n}\right)_{n \in \mathbb{N}}$ is a family of self-mappings of $X$ satisfying property $\left(P_{2}\right)$ and having common fixed points. Set

$$
F:=\bigcap_{n \in \mathbb{N}} \operatorname{Fix}\left(T_{n}\right) \neq \emptyset .
$$

For $x \in X$, we define the following iteration starting with $x$ :

$$
x_{0}:=x, \quad x_{n+1}:=T_{n} x_{n} \text { for all } n \in \mathbb{N} .
$$

Let $\left(\gamma_{n}\right)$ be a sequence of positive real numbers such that $\sum_{n=0}^{\infty} \gamma_{n}^{2}=\infty$.

The following conditions will also be considered in the sequel:

(C1) for all $n, m \in \mathbb{N}$ and $w \in X, d\left(T_{n} w, T_{m} w\right) \leq \frac{\left|\gamma_{n}-\gamma_{m}\right|}{\gamma_{n}} d\left(w, T_{n} w\right)$;

$(C 2)$ the sequence $\left(\frac{d\left(x_{n}, x_{n+1}\right)}{\gamma_{n}}\right)_{n \in \mathbb{N}}$ is nonincreasing.

We include below a series of preliminary results.

Lemma 3.1. Suppose that $(C 1)$ holds. Then Fix $\left(T_{n}\right)=F$ for every $n \in \mathbb{N}$.

Proof. It follows immediately.

Lemma 3.2. For all $p \in F$ and all $n \in \mathbb{N}$, we have that

$$
d^{2}\left(x_{n+1}, p\right) \leq d^{2}\left(x_{n}, p\right)-d^{2}\left(x_{n}, x_{n+1}\right) .
$$

In particular, $\left(x_{n}\right)$ is Fejér monotone with respect to $F$.

Proof. Let $p \in F$ and $n \in \mathbb{N}$. Since $T_{n}$ satisfies property $\left(P_{2}\right)$ and $p \in F i x\left(T_{n}\right)$, we have that $2 d^{2}\left(T_{n} x_{n}, p\right) \leq d^{2}\left(x_{n}, p\right)+d^{2}\left(T_{n} x_{n}, p\right)-d^{2}\left(x_{n}, T_{n} x_{n}\right)$. It follows that $d^{2}\left(T_{n} x_{n}, p\right) \leq d^{2}\left(x_{n}, p\right)-$ $d^{2}\left(x_{n}, T_{n} x_{n}\right)$, hence the conclusion.

Lemma 3.3. Assume that $(C 2)$ is satisfied. Then

$$
\lim _{n \rightarrow \infty} d\left(x_{n}, x_{n+1}\right)=0 \quad \text { and } \quad \lim _{n \rightarrow \infty} \frac{d\left(x_{n}, x_{n+1}\right)}{\gamma_{n}}=0 .
$$

Proof. Since $F \neq \emptyset$, there exists $p \in F$. Let $b>0$ be such that $d(x, p) \leq b$. For every $n \in \mathbb{N}$, we have, by Lemma 3.2, that

$$
\sum_{k=0}^{n} d^{2}\left(x_{k}, x_{k+1}\right) \leq \sum_{k=0}^{n}\left(d^{2}\left(x_{k}, p\right)-d^{2}\left(x_{k+1}, p\right)\right)=d^{2}(x, p)-d^{2}\left(x_{n+1}, p\right) \leq b^{2} .
$$

It follows that the series $\sum_{n=0}^{\infty} d^{2}\left(x_{n}, x_{n+1}\right)$ converges, so $\lim _{n \rightarrow \infty} d\left(x_{n}, x_{n+1}\right)=0$.

We prove now that $\lim _{n \rightarrow \infty} \frac{d\left(x_{n}, x_{n+1}\right)}{\gamma_{n}}=0$. Let $\varepsilon>0$. Since $\sum_{n=0}^{\infty} \gamma_{n}^{2}=\infty$, there exists $N \in \mathbb{N}$ such that $\sum_{k=0}^{N} \gamma_{k}^{2} \geq b^{2} / \varepsilon^{2}$. If for all $k \in\{0, \ldots, N\}$ one has that $\frac{d\left(x_{k}, x_{k+1}\right)}{\gamma_{k}}>\varepsilon$, we get that

$$
\sum_{k=0}^{N} d^{2}\left(x_{k}, x_{k+1}\right)>\sum_{k=0}^{N} \gamma_{k}^{2} \varepsilon^{2} \geq b^{2},
$$

a contradiction. Hence, there exists $M \in\{0, \ldots, N\}$ such that $\frac{d\left(x_{M}, x_{M+1}\right)}{\gamma_{M}} \leq \varepsilon$. Since, by $(C 2)$, the sequence $\left(\frac{d\left(x_{n}, x_{n+1}\right)}{\gamma_{n}}\right)$ is nonincreasing, we get that $\frac{d\left(x_{n}, x_{n+1}\right)}{\gamma_{n}} \leq \varepsilon$ for all $n \geq M$. 
Proposition 3.4. Assume that $(C 1)$ and $(C 2)$ are satisfied. Then for all $m \in \mathbb{N}$,

$$
\lim _{n \rightarrow \infty} d\left(x_{n}, T_{m} x_{n}\right)=0 .
$$

Proof. Let $m \in \mathbb{N}$. We get that for all $n \in \mathbb{N}$,

$$
\begin{aligned}
d\left(x_{n}, T_{m} x_{n}\right) & \leq d\left(x_{n}, x_{n+1}\right)+d\left(x_{n+1}, T_{m} x_{n}\right)=d\left(x_{n}, x_{n+1}\right)+d\left(T_{n} x_{n}, T_{m} x_{n}\right) \\
& \leq d\left(x_{n}, x_{n+1}\right)+\frac{\left|\gamma_{n}-\gamma_{m}\right|}{\gamma_{n}} d\left(x_{n}, T_{n} x_{n}\right) \quad \text { by }(C 1) \\
& \leq 2 d\left(x_{n}, x_{n+1}\right)+\gamma_{m} \cdot \frac{d\left(x_{n}, x_{n+1}\right)}{\gamma_{n}} .
\end{aligned}
$$

Our conclusion follows by applying Lemma 3.3.

We can prove now the main result of this section.

Theorem 3.5 (Abstract Proximal Point Algorithm). Let $X$ be a complete CAT $(0)$ space and $\left(T_{n}\right)$ be a family of self-mappings of $X$ satisfying property $\left(P_{2}\right)$ and having common fixed points. Set $F:=\bigcap_{n \in \mathbb{N}} F i x\left(T_{n}\right) \neq \emptyset$. For $x \in X$, let $\left(x_{n}\right)$ be defined by (8). Let $\left(\gamma_{n}\right)$ be a sequence of positive real numbers such that $\sum_{n=0}^{\infty} \gamma_{n}^{2}=\infty$. Assume that $(C 1)$ and $(C 2)$ hold.

Then $\left(x_{n}\right) \Delta$-converges to a point in $F$.

Proof. Note first that by Lemma 3.2, $\left(x_{n}\right)$ is Fejér monotone with respect to $F$, hence bounded. Let $\left(u_{n}\right)$ be an arbitrary subsequence of $\left(x_{n}\right)$. By Proposition $2.5,\left(u_{n}\right)$ has a unique asymptotic center $u$. We shall prove that $u \in F$, so let $m \in \mathbb{N}$ be arbitrary. Note that

$$
d\left(T_{m} u, u_{n}\right) \leq d\left(T_{m} u, T_{m} u_{n}\right)+d\left(u_{n}, T_{m} u_{n}\right) \leq d\left(u, u_{n}\right)+d\left(u_{n}, T_{m} u_{n}\right) .
$$

Applying Lemma 2.4 with $\alpha_{n}=1, \beta_{n}=d\left(u_{n}, T_{m} u_{n}\right), p=N=0$ and using the fact that $\lim _{n \rightarrow \infty} d\left(u_{n}, T_{m} u_{n}\right)=0$ (by Proposition 3.4), we get that $T_{m} u=u$.

Finally, Proposition 2.8 yields that $\left(x_{n}\right) \Delta$-converges to a point in $F$.

\subsection{Jointly firmly nonexpansive families of mappings}

We shall now proceed to the second stage of our abstraction - that is, giving a natural condition for a family $\left(T_{n}\right)$ and a sequence $\left(\gamma_{n}\right)$ of positive numbers such that the previous general conditions are satisfied. This can be regarded as an extension of the project initiated in [1] with the definition and the asymptotic behaviour of a firmly nonexpansive mapping to the case of a countable family of mappings. Recall that the notion of a firmly nonexpansive mapping in a Hilbert space has two analogues when considered within the more general setting of $\operatorname{CAT}(0)$ spaces. In the same spirit, we shall present here two definitions that apply to families of mappings which coincide when restricted to Hilbert spaces.

In the sequel, $X$ is a $\operatorname{CAT}(0)$ space, $T_{n}: X \rightarrow X$ for every $n \in \mathbb{N}$ and $\left(\gamma_{n}\right)$ is a sequence of positive real numbers.

Definition 3.6. The family $\left(T_{n}\right)$ is said to be jointly firmly nonexpansive with respect to $\left(\gamma_{n}\right)$ if for all $n, m \in \mathbb{N}, x, y \in X$ and all $\alpha, \beta \in[0,1]$ such that $(1-\alpha) \gamma_{n}=(1-\beta) \gamma_{m}$,

$$
d\left(T_{n} x, T_{m} y\right) \leq d\left((1-\alpha) x+\alpha T_{n} x,(1-\beta) y+\beta T_{m} y\right) .
$$

Definition 3.7. We say that the family $\left(T_{n}\right)$ is jointly $\left(P_{2}\right)$ with respect to $\left(\gamma_{n}\right)$ if for all $n, m \in \mathbb{N}$ and all $x, y \in X$,

$$
\frac{1}{\gamma_{m}}\left(d^{2}\left(T_{n} x, T_{m} y\right)+d^{2}\left(y, T_{m} y\right)-d^{2}\left(y, T_{n} x\right)\right) \leq \frac{1}{\gamma_{n}}\left(d^{2}\left(x, T_{m} y\right)-d^{2}\left(x, T_{n} x\right)-d^{2}\left(T_{n} x, T_{m} y\right)\right) .
$$

Lemma 3.8. If $\left(T_{n}\right)$ is jointly firmly nonexpansive (resp. jointly $\left(P_{2}\right)$ ) with respect to $\left(\gamma_{n}\right)$, then each $T_{n}$ is firmly nonexpansive (resp. satisfies property $\left(P_{2}\right)$ ). 
Proof. Apply (9) (resp. (10)) for $m=n$. In the first case, remark that given $t \in[0,1]$, we take $\alpha=\beta=t$.

Proposition 3.9. If $\left(T_{n}\right)$ is jointly firmly nonexpansive with respect to $\left(\gamma_{n}\right)$, then $\left(T_{n}\right)$ is jointly $\left(P_{2}\right)$ with respect to $\left(\gamma_{n}\right)$.

Proof. Let $m, n \in \mathbb{N}$ and $x, y \in X$. We choose arbitrarily $\alpha \in\left(1-\min \left\{\gamma_{m} / \gamma_{n}, 1\right\}, 1\right)$ and set

$$
\beta:=1-(1-\alpha) \frac{\gamma_{n}}{\gamma_{m}}
$$

Then $\beta \in(0,1)$ and $(1-\alpha) \gamma_{n}=(1-\beta) \gamma_{m}$. Hence, applying the fact that $\left(T_{n}\right)$ is jointly firmly nonexpansive and the inequality (1) twice, we get that

$$
\begin{aligned}
d^{2}\left(T_{n} x, T_{m} y\right) \leq & d^{2}\left((1-\alpha) x+\alpha T_{n} x,(1-\beta) y+\beta T_{m} y\right) \\
\leq & (1-\alpha) d^{2}\left(x,(1-\beta) y+\beta T_{m} y\right)+\alpha d^{2}\left(T_{n} x,(1-\beta) y+\beta T_{m} y\right)-\alpha(1-\alpha) d^{2}\left(x, T_{n} x\right) \\
\leq & (1-\alpha)(1-\beta) d^{2}(x, y)+(1-\alpha) \beta d^{2}\left(x, T_{m} y\right)-(1-\alpha) \beta(1-\beta) d^{2}\left(y, T_{m} y\right)+ \\
& +\alpha(1-\beta) d^{2}\left(T_{n} x, y\right)+\alpha \beta d^{2}\left(T_{n} x, T_{m} y\right)-\alpha \beta(1-\beta) d^{2}\left(y, T_{m} y\right)-\alpha(1-\alpha) d^{2}\left(x, T_{n} x\right) \\
= & (1-\alpha)(1-\beta) d^{2}(x, y)+(1-\beta) \alpha d^{2}\left(T_{n} x, y\right)+(1-\alpha) \beta d^{2}\left(x, T_{m} y\right)+ \\
& +\alpha \beta d^{2}\left(T_{n} x, T_{m} y\right)-\alpha(1-\alpha) d^{2}\left(x, T_{n} x\right)-\beta(1-\beta) d^{2}\left(y, T_{m} y\right),
\end{aligned}
$$

so

$$
\begin{aligned}
(1-\alpha \beta) d^{2}\left(T_{n} x, T_{m} y\right) \leq & (1-\alpha)(1-\beta) d^{2}(x, y)+(1-\beta) \alpha d^{2}\left(T_{n} x, y\right)+(1-\alpha) \beta d^{2}\left(x, T_{m} y\right)- \\
& -\alpha(1-\alpha) d^{2}\left(x, T_{n} x\right)-\beta(1-\beta) d^{2}\left(y, T_{m} y\right) .
\end{aligned}
$$

Dividing now the above inequality by $1-\alpha>0$, we obtain that

$$
\begin{aligned}
\frac{1-\alpha \beta}{1-\alpha} d^{2}\left(T_{n} x, T_{m} y\right) \leq & (1-\beta) d^{2}(x, y)+\frac{(1-\beta) \alpha}{1-\alpha} d^{2}\left(T_{n} x, y\right)+\beta d^{2}\left(x, T_{m} y\right)- \\
& -\alpha d^{2}\left(x, T_{n} x\right)-\frac{\beta(1-\beta)}{1-\alpha} d^{2}\left(y, T_{m} y\right) .
\end{aligned}
$$

By easy computations, one can see that

$$
\frac{1-\alpha \beta}{1-\alpha}=1+\alpha \frac{\gamma_{n}}{\gamma_{m}}, \quad \frac{(1-\beta) \alpha}{1-\alpha}=\alpha \frac{\gamma_{n}}{\gamma_{m}} \quad \text { and } \quad \frac{\beta(1-\beta)}{1-\alpha}=\left(1-(1-\alpha) \frac{\gamma_{n}}{\gamma_{m}}\right) \frac{\gamma_{n}}{\gamma_{m}} .
$$

Therefore, we have that

$$
\begin{aligned}
\left(1+\alpha \frac{\gamma_{n}}{\gamma_{m}}\right) d^{2}\left(T_{n} x, T_{m} y\right) \leq & (1-\alpha) \frac{\gamma_{n}}{\gamma_{m}} d^{2}(x, y)+\alpha \frac{\gamma_{n}}{\gamma_{m}} d^{2}\left(T_{n} x, y\right)+\left(1-(1-\alpha) \frac{\gamma_{n}}{\gamma_{m}}\right) d^{2}\left(x, T_{m} y\right)- \\
& -\alpha d^{2}\left(x, T_{n} x\right)-\left(1-(1-\alpha) \frac{\gamma_{n}}{\gamma_{m}}\right) \frac{\gamma_{n}}{\gamma_{m}} d^{2}\left(y, T_{m} y\right) .
\end{aligned}
$$

Letting $\alpha \rightarrow 1$, we get that

$$
\left(1+\frac{\gamma_{n}}{\gamma_{m}}\right) d^{2}\left(T_{n} x, T_{m} y\right) \leq \frac{\gamma_{n}}{\gamma_{m}} d^{2}\left(T_{n} x, y\right)+d^{2}\left(x, T_{m} y\right)-d^{2}\left(x, T_{n} x\right)-\frac{\gamma_{n}}{\gamma_{m}} d^{2}\left(y, T_{m} y\right),
$$

so

$$
\frac{\gamma_{n}}{\gamma_{m}}\left(d^{2}\left(T_{n} x, T_{m} y\right)+d^{2}\left(y, T_{m} y\right)-d^{2}\left(T_{n} x, y\right)\right) \leq d^{2}\left(x, T_{m} y\right)-d^{2}\left(x, T_{n} x\right)-d^{2}\left(T_{n} x, T_{m} y\right) .
$$

Divide by $\gamma_{n}$ to obtain (10), our required inequality. 
Using the quasi-linearization function defined by $(3)$, the joint $\left(P_{2}\right)$ condition can equivalently be expressed as:

$$
\frac{1}{\gamma_{m}}\left\langle\overrightarrow{T_{n} x T_{m} y}, \overrightarrow{y T_{m} y}\right\rangle \leq \frac{1}{\gamma_{n}}\left\langle\overrightarrow{T_{n} x T_{m} y}, \overrightarrow{x T_{n} x}\right\rangle,
$$

for all $n, m \in \mathbb{N}$.

Proposition 3.10. Suppose that $\left(T_{n}\right)$ is jointly $\left(P_{2}\right)$ with respect to $\left(\gamma_{n}\right)$. Then for all $m, n \in \mathbb{N}$ and all $w \in X$,

$$
d\left(T_{n} w, T_{m} w\right) \leq \frac{\left|\gamma_{n}-\gamma_{m}\right|}{\gamma_{n}} d\left(w, T_{n} w\right) .
$$

Proof. Let $m, n \in \mathbb{N}$. We shall denote, for simplicity, $T:=T_{n}, U:=T_{m}, \lambda:=\gamma_{n}, \mu:=\gamma_{m}$.

We want to show that for all $w \in X$,

$$
d(T w, U w) \leq \frac{|\lambda-\mu|}{\lambda} d(w, T w) .
$$

If $T w=U w$, the statement is trivially true. Let $w \in X$ be such that $T w \neq U w$.

Claim: $(\lambda+\mu) d^{2}(T w, U w) \leq(\lambda-\mu)\left(d^{2}(w, T w)-d^{2}(w, U w)\right)$.

Proof of claim: We have that

$$
\frac{1}{\mu}\langle\overrightarrow{T w U w}, \overrightarrow{w U w}\rangle \leq \frac{1}{\lambda}\langle\overrightarrow{T w U w}, \overrightarrow{w T w}\rangle,
$$

and, by multiplying with $(-\lambda)$, we get that

$$
\langle\overrightarrow{T w U w}, \overrightarrow{T w w}\rangle \leq \frac{\lambda}{\mu}\langle\overrightarrow{T w U w}, \overrightarrow{U w w}\rangle .
$$

A simple expansion of $\langle\cdot, \cdot\rangle$ shows that

$$
d^{2}(T w, U w)=d^{2}(w, U w)-d^{2}(w, T w)+2\langle\overrightarrow{T w U w}, \overrightarrow{T w w}\rangle .
$$

By exchanging the roles of $T$ and $U$ in the above equation, we obtain that

$$
d^{2}(U w, T w)=d^{2}(w, T w)-d^{2}(w, U w)+2\langle\overrightarrow{U w T w}, \overrightarrow{U w w}\rangle .
$$

Applying (12) and (13) and multiplying (14) by $\frac{\lambda}{\mu}$, we get that

$$
\begin{aligned}
d^{2}(T w, U w) & \leq d^{2}(w, U w)-d^{2}(w, T w)+\frac{2 \lambda}{\mu}\langle\overrightarrow{T w U w}, \overrightarrow{U w w}\rangle, \\
\frac{\lambda}{\mu} d^{2}(U w, T w) & =\frac{\lambda}{\mu} d^{2}(w, T w)-\frac{\lambda}{\mu} d^{2}(w, U w)+\frac{2 \lambda}{\mu}\langle\overrightarrow{U w T w}, \overrightarrow{U w w}\rangle .
\end{aligned}
$$

As a consequence, it follows that

$$
\left(1+\frac{\lambda}{\mu}\right) d^{2}(T w, U w) \leq\left(\frac{\lambda}{\mu}-1\right)\left(d^{2}(w, T w)-d^{2}(w, U w)\right) .
$$

Multiply by $\mu$ to get the claim.

We distinguish now two cases, according to the sign of $\lambda-\mu$.

When $\lambda-\mu$ is negative, we obtain, using the claim, that

$$
\begin{aligned}
(\lambda+\mu) d^{2}(T w, U w) & \leq(\lambda-\mu)\left(d^{2}(w, T w)-d^{2}(w, U w)\right) \\
& =(\mu-\lambda)\left(d^{2}(w, U w)-d^{2}(w, T w)\right) \\
& \leq(\mu-\lambda)\left((d(w, T w)+d(T w, U w))^{2}-d^{2}(w, T w)\right) \\
& =(\mu-\lambda) d(T w, U w)(d(T w, U w)+2 d(w, T w)) .
\end{aligned}
$$


Dividing by $d(T w, U w) \neq 0$, we have that

$$
(\lambda+\mu) d(T w, U w) \leq 2(\mu-\lambda) d(w, T w)+(\mu-\lambda) d(T w, U w),
$$

so

$$
2 \lambda d(T w, U w) \leq 2(\mu-\lambda) d(w, T w) .
$$

Thus,

$$
d(T w, U w) \leq \frac{\mu-\lambda}{\lambda} d(w, T w)=\frac{|\lambda-\mu|}{\lambda} d(w, T w)
$$

as required.

Now, when $\lambda-\mu$ is positive, we proceed as follows. By the reverse triangle inequality for metric spaces, we have that

$$
d^{2}(w, U w) \geq|d(T w, U w)-d(w, T w)|^{2}=d^{2}(T w, U w)-2 d(w, T w) d(T w, U w)+d^{2}(w, T w) .
$$

Applying the claim, we obtain that

$$
\begin{aligned}
(\lambda+\mu) d^{2}(T w, U w) & \leq(\lambda-\mu)\left(d^{2}(w, T w)-d^{2}(w, U w)\right) \\
& \leq(\lambda-\mu)\left(2 d(w, T w) d(T w, U w)-d^{2}(T w, U w)\right) \\
& =(\lambda-\mu) d(T w, U w)(2 d(w, T w)-d(T w, U w)) .
\end{aligned}
$$

As above, one gets that

$$
d(T w, U w) \leq \frac{\lambda-\mu}{\lambda} d(w, T w)=\frac{|\lambda-\mu|}{\lambda} d(w, T w)
$$

Corollary 3.11. Suppose that $\left(T_{n}\right)$ is jointly $\left(P_{2}\right)$ with respect to $\left(\gamma_{n}\right)$. Then any two mappings of the family have the same set of fixed points.

Proof. It follows from Proposition 3.10 and Lemma 3.1.

Proposition 3.12. Assume that $\left(T_{n}\right)$ is jointly $\left(P_{2}\right)$ with respect to $\left(\gamma_{n}\right)$. Let $x \in X$ and $\left(x_{n}\right)$ be given by (8). Then the sequence $\left(\frac{d\left(x_{n}, x_{n+1}\right)}{\gamma_{n}}\right)$ is nonincreasing.

Proof. Let $n \in \mathbb{N}$. By (11),

$$
\frac{1}{\gamma_{n+1}}\left\langle\overrightarrow{T_{n} x_{n} T_{n+1} x_{n+1}}, \overrightarrow{x_{n+1} T_{n+1} x_{n+1}}\right\rangle \leq \frac{1}{\gamma_{n}}\left\langle\overrightarrow{T_{n} x_{n} T_{n+1} x_{n+1}}, \overrightarrow{x_{n} T_{n} x_{n}}\right\rangle,
$$

that is

$$
\frac{1}{\gamma_{n+1}}\left\langle\overrightarrow{x_{n+1} x_{n+2}}, \overrightarrow{x_{n+1} x_{n+2}}\right\rangle \leq \frac{1}{\gamma_{n}}\left\langle\overrightarrow{x_{n+1} x_{n+2}}, \overrightarrow{x_{n} x_{n+1}}\right\rangle
$$

Thus,

$$
\begin{aligned}
0 & \leq \frac{1}{\gamma_{n}}\left\langle\overrightarrow{x_{n+1} x_{n+2}}, \overrightarrow{x_{n} x_{n+1}}\right\rangle-\frac{d^{2}\left(x_{n+1}, x_{n+2}\right)}{\gamma_{n+1}} \\
& =\gamma_{n+1}\left(\frac{1}{\gamma_{n} \gamma_{n+1}}\left\langle\overrightarrow{x_{n+1} x_{n+2}}, \overrightarrow{x_{n} x_{n+1}}\right\rangle-\frac{d^{2}\left(x_{n+1}, x_{n+2}\right)}{\gamma_{n+1}^{2}}\right) \\
& \leq \gamma_{n+1}\left(\frac{d\left(x_{n}, x_{n+1}\right)}{\gamma_{n}} \cdot \frac{d\left(x_{n+1}, x_{n+2}\right)}{\gamma_{n+1}}-\frac{d^{2}\left(x_{n+1}, x_{n+2}\right)}{\gamma_{n+1}^{2}}\right) \quad \text { by }(5) \\
& =\gamma_{n+1} \cdot \frac{d\left(x_{n+1}, x_{n+2}\right)}{\gamma_{n+1}}\left(\frac{d\left(x_{n}, x_{n+1}\right)}{\gamma_{n}}-\frac{d\left(x_{n+1}, x_{n+2}\right)}{\gamma_{n+1}}\right) .
\end{aligned}
$$

It follows that $\frac{d\left(x_{n+1}, x_{n+2}\right)}{\gamma_{n+1}} \leq \frac{d\left(x_{n}, x_{n+1}\right)}{\gamma_{n}}$. 
We give now another abstract version of the Proximal Point Algorithm.

Theorem 3.13. Let $X$ be a complete $C A T(0)$ space, $T_{n}: X \rightarrow X$ for every $n \in \mathbb{N}$ and $\left(\gamma_{n}\right)$ be a sequence of positive real numbers satisfying $\sum_{n=0}^{\infty} \gamma_{n}^{2}=\infty$. Assume that the family $\left(T_{n}\right)$ is jointly $\left(P_{2}\right)$ with respect to $\left(\gamma_{n}\right)$ (in particular, $\left(T_{n}\right)$ may be jointly firmly nonexpansive) and that $F:=\bigcap_{n \in \mathbb{N}} \operatorname{Fix}\left(T_{n}\right) \neq \emptyset$. Let $x \in X$ and $\left(x_{n}\right)$ be given by (8).

Then $\left(x_{n}\right) \Delta$-converges to a point in $F$.

Proof. By Lemma 3.8, each $T_{n}$ satisfies property $\left(P_{2}\right)$. We can now apply Theorem 3.5, as conditions $(C 1)$ and $(C 2)$ follow from Propositions 3.10 and 3.12 , respectively.

\subsubsection{The case of Hilbert spaces}

Assume now that $H$ is a Hilbert space with inner product $\langle\cdot, \cdot\rangle$. We show next that joint firm nonexpansivity coincides with the joint $\left(P_{2}\right)$ condition.

Proposition 3.14. Let $\left(T_{n}\right)$ be a family of self-mappings of $H$ and $\left(\gamma_{n}\right)$ be a sequence of positive real numbers. Then $\left(T_{n}\right)$ is jointly $\left(P_{2}\right)$ with respect to $\left(\gamma_{n}\right)$ if and only if $\left(T_{n}\right)$ is jointly firmly nonexpansive with respect to $\left(\gamma_{n}\right)$.

Proof. " $\Leftarrow$ " By Proposition 3.9.

" $\Rightarrow$ " Let $m, n \in \mathbb{N}, x, y \in H$ and $\alpha, \beta \in[0,1]$ be such that $(1-\alpha) \gamma_{n}=(1-\beta) \gamma_{m}=: \delta$. A simple computation yields the following two identities

$$
(1-\alpha) x+\alpha T_{n} x=T_{n} x+\frac{\delta}{\gamma_{n}}\left(x-T_{n} x\right) \quad \text { and } \quad(1-\beta) y+\beta T_{m} y=T_{m} y+\frac{\delta}{\gamma_{m}}\left(y-T_{m} y\right) .
$$

It follows that

$$
\begin{aligned}
\left\|\left((1-\alpha) x+\alpha T_{n} x\right)-\left((1-\beta) y+\beta T_{m} y\right)\right\|^{2}= & \left\|\left(T_{n} x-T_{m} y\right)+\left(\frac{\delta}{\gamma_{n}}\left(x-T_{n} x\right)-\frac{\delta}{\gamma_{m}}\left(y-T_{m} y\right)\right)\right\|^{2} \\
= & \left\|T_{n} x-T_{m} y\right\|^{2}+\delta^{2}\left\|\frac{1}{\gamma_{n}}\left(x-T_{n} x\right)-\frac{1}{\gamma_{m}}\left(y-T_{m} y\right)\right\|^{2} \\
& +2 \delta\left\langle T_{n} x-T_{m} y, \frac{1}{\gamma_{n}}\left(x-T_{n} x\right)-\frac{1}{\gamma_{m}}\left(y-T_{m} y\right)\right\rangle .
\end{aligned}
$$

In order to show that the right-hand side is greater than or equal to $\left\|T_{n} x-T_{m} y\right\|^{2}$, which is what we are aiming to prove here, it is sufficient to show that

$$
D:=\left\langle T_{n} x-T_{m} y, \frac{1}{\gamma_{n}}\left(x-T_{n} x\right)-\frac{1}{\gamma_{m}}\left(y-T_{m} y\right)\right\rangle \geq 0 .
$$

Remark that

$$
\begin{aligned}
D & =\frac{1}{\gamma_{n}}\left\langle T_{n} x-T_{m} y, x-T_{n} x\right\rangle-\frac{1}{\gamma_{m}}\left\langle T_{n} x-T_{m} y, y-T_{m} y\right\rangle \\
& =\frac{1}{\gamma_{n}}\left\langle\overrightarrow{T_{n} x T_{m} y}, \overrightarrow{x T_{n} x}\right\rangle-\frac{1}{\gamma_{m}}\left\langle\overrightarrow{T_{n} x T_{m} y}, \overrightarrow{y T_{m} y}\right\rangle \quad \text { by }(4) \\
& \geq 0 \quad \text { by (11). }
\end{aligned}
$$

Thus, $\left(T_{n}\right)$ is jointly firmly nonexpansive with respect to $\left(\gamma_{n}\right)$.

Since $\Delta$-convergence coincides with weak convergence in Hilbert spaces, we get, as an immediate consequence of Theorem 3.13, the following abstract version of the Proximal Point Algorithm.

Theorem 3.15. Let $H$ be a Hilbert space, $T_{n}: H \rightarrow H$ for every $n \in \mathbb{N}$ and $\left(\gamma_{n}\right)$ be a sequence of positive real numbers satisfying $\sum_{n=0}^{\infty} \gamma_{n}^{2}=\infty$. Assume that the family $\left(T_{n}\right)$ is jointly firmly nonexpansive with respect to $\left(\gamma_{n}\right)$ and that $F:=\bigcap_{n \in \mathbb{N}} F i x\left(T_{n}\right) \neq \emptyset$. Let $x \in H$ and $\left(x_{n}\right)$ be given by (8).

Then $\left(x_{n}\right)$ converges weakly to a point in $F$. 
We are now in a position to prove that specific instances of the proximal point algorithm satisfy the stronger requirement that their associated families of resolvents are jointly firmly nonexpansive, thus justifying our choice of definitions. Three concrete problems - minimizing convex functions, finding fixed points of nonexpansive mappings and finding zeros of maximally monotone operators - are used to illustrate this fact. We may then apply Theorems 3.13 and 3.15 in order to obtain classical weak convergence results for these iterations.

\subsection{Minimizers of convex proper lsc functions}

In the sequel, $X$ is a complete $\operatorname{CAT}(0)$ space and $f: X \rightarrow(-\infty, \infty]$ is a convex, proper, lower semicontinuous (lsc) function. A point $x \in X$ is said to be a minimizer of $f$ if $f(x)=\inf _{y \in X} f(y)$. The set of minimizers of $f$ is denoted by $\operatorname{Argmin}(f)$.

For any $\gamma>0$, let us denote, following [3],

$$
J_{\gamma}: X \rightarrow X, \quad J_{\gamma}(x):=\operatorname{argmin}_{y \in X}\left[f(y)+\frac{1}{2 \gamma} d^{2}(x, y)\right] .
$$

The mapping $J_{\gamma}$, defined in the context of CAT(0) spaces by Jost [25], is called the (MoreauYosida) resolvent or the proximal mapping of $f$ of order $\gamma$.

We recall in the following proposition some well-known properties proven in [25].

Proposition 3.16. Let $\gamma>0$. Then

(i) $\operatorname{Fix}\left(J_{\gamma}\right)=\operatorname{Argmin}(f)$.

(ii) $J_{\gamma}$ is nonexpansive.

(iii) For all $x \in X$ and all $t \in[0,1]$, the following holds:

$$
J_{(1-t) \gamma}\left((1-t) x+t J_{\gamma}(x)\right)=J_{\gamma}(x) .
$$

Proposition 3.17. Let $\left(\gamma_{n}\right)$ be a sequence of positive real numbers. Then the family $\left(J_{\gamma_{n}}\right)$ is jointly firmly nonexpansive with respect to $\left(\gamma_{n}\right)$.

Proof. Let $m, n \in \mathbb{N}, x, y \in X$ and $\alpha, \beta \in[0,1]$ be such that $(1-\alpha) \gamma_{n}=(1-\beta) \gamma_{m}=: \delta$. Applying Proposition 3.16, we get that

$$
\begin{aligned}
d\left(J_{\gamma_{n}} x, J_{\gamma_{m}} y\right) & =d\left(J_{(1-\alpha) \gamma_{n}}\left((1-\alpha) x+\alpha J_{\gamma_{n}} x\right), J_{(1-\beta) \gamma_{m}}\left((1-\beta) y+\beta J_{\gamma_{m}} y\right)\right) \\
& =d\left(J_{\delta}\left((1-\alpha) x+\alpha J_{\gamma_{n}} x\right), J_{\delta}\left((1-\beta) y+\beta J_{\gamma_{m}} y\right)\right) \\
& \leq d\left((1-\alpha) x+\alpha J_{\gamma_{n}} x,(1-\beta) y+\beta J_{\gamma_{m}} y\right) .
\end{aligned}
$$

As a consequence of Theorem 3.13, we get the following $\Delta$-convergence result.

Theorem 3.18. Assume that Argmin $(f) \neq \emptyset$ and let $\left(\gamma_{n}\right)$ be a sequence of positive real numbers such that $\sum_{n=0}^{\infty} \gamma_{n}^{2}=\infty$. For any $x \in X$, define the sequence $\left(x_{n}\right)$, starting with $x$, by

$$
x_{0}:=x, \quad x_{n+1}:=J_{\gamma_{n}} x_{n} \text { for all } n \in \mathbb{N} .
$$

Then $\left(x_{n}\right) \Delta$-converges to a minimizer of $f$.

Proof. For all $n \in \mathbb{N}$, put $T_{n}:=J_{\gamma_{n}}$. By Proposition 3.16.(i), Fix $\left(T_{n}\right)=\operatorname{Argmin}(f)$ for all $n \in \mathbb{N}$. Furthermore, by Proposition 3.17 , the family $\left(T_{n}\right)$ is jointly firmly nonexpansive with respect to $\left(\gamma_{n}\right)$. Hence, we may apply Theorem 3.13 to derive our conclusion.

The above theorem is a slightly weaker variant (with a completely different proof) of a result due to Bačák [3, Theorem 1.4], since one uses here the stronger assumption $\sum_{n=0}^{\infty} \gamma_{n}^{2}=\infty$ instead of $\sum_{n=0}^{\infty} \gamma_{n}=\infty$. We point out that an analysis of Bačák's original statement from the point of view of proof mining was previously carried out in [41, 42]. 


\subsection{Fixed points of nonexpansive mappings}

We proceed now to give another application. Let $X$ be a complete CAT(0) space and $T: X \rightarrow X$ be a nonexpansive mapping.

For $x \in X$ and $\gamma>0$ we define

$$
G_{T, x, \gamma}: X \rightarrow X, \quad G_{T, x, \gamma}(y):=\frac{1}{1+\gamma} x+\frac{\gamma}{1+\gamma} T y .
$$

It is easy to see that this mapping is Lipschitz with constant $\frac{\gamma}{1+\gamma} \in(0,1)$. Therefore it admits a unique fixed point, which we shall denote by $R_{T, \gamma} x$. We have thus defined a mapping $R_{T, \gamma}: X \rightarrow$ $X$, called the resolvent of order $\gamma$ of $T$, which satisfies, for any $x \in X$,

$$
R_{T, \gamma} x=\frac{1}{1+\gamma} x+\frac{\gamma}{1+\gamma} T R_{T, \gamma} x
$$

We immediately obtain that $\operatorname{Fix}\left(R_{T, \gamma}\right)=\operatorname{Fix}(T)$ for all $\gamma>0$.

Proposition 3.19. Let $\left(\gamma_{n}\right)$ be a sequence of positive real numbers. Then the family $\left(R_{T, \gamma_{n}}\right)$ is jointly firmly nonexpansive with respect to $\left(\gamma_{n}\right)$.

Proof. Let $m, n \in \mathbb{N}, x, y \in X$ and $\alpha, \beta \in[0,1]$ be such that $(1-\alpha) \gamma_{n}=(1-\beta) \gamma_{m}=: \delta$. Denote

$$
u:=(1-\alpha) x+\alpha R_{T, \gamma_{n}} x, \quad v:=(1-\beta) y+\beta R_{T, \gamma_{m}} y .
$$

Then we have to show that

$$
d\left(R_{T, \gamma_{n}} x, R_{T, \gamma_{m}} y\right) \leq d(u, v) .
$$

Using (16) and the definition of $u$, we may apply [1, Lemma 2.4.(iii)] to obtain that

$$
R_{T, \gamma_{n}} x=(1-\nu) u+\nu T R_{T, \gamma_{n}} x,
$$

where

$$
\nu:=\frac{(1-\alpha) \frac{\gamma_{n}}{1+\gamma_{n}}}{1-\alpha \cdot \frac{\gamma_{n}}{1+\gamma_{n}}}=\frac{\delta}{1+\delta} .
$$

We remark that $\nu \neq 1$. Also note that, while the statement of [1, Lemma 2.4.(iii)] requires the four points to be pairwise distinct, its conclusion is trivial to show in the case of some of them are equal. We show similarly that

$$
R_{T, \gamma_{m}} y=(1-\nu) v+\nu T R_{T, \gamma_{m}} y .
$$

Applying (2) and the nonexpansivity of $T$, we get that

$$
\begin{aligned}
d\left(R_{T, \gamma_{n}} x, R_{T, \gamma_{m}} y\right) & =d\left((1-\nu) u+\nu T R_{T, \gamma_{n}} x,(1-\nu) v+\nu T R_{T, \gamma_{m}} y\right) \\
& \leq(1-\nu) d(u, v)+\nu d\left(T R_{T, \gamma_{n}} x, T R_{T, \gamma_{m}} y\right) \\
& \leq(1-\nu) d(u, v)+\nu d\left(R_{T, \gamma_{n}} x, R_{T, \gamma_{m}} y\right) .
\end{aligned}
$$

It follows immediately that (17) holds.

As an immediate application of Theorem 3.13, we get

Theorem 3.20. Assume that Fix $(T) \neq \emptyset$ and let $\left(\gamma_{n}\right)$ be a sequence of positive real numbers such that $\sum_{n=0}^{\infty} \gamma_{n}^{2}=\infty$. For any $x \in X$, define the sequence $\left(x_{n}\right)$ by

$$
x_{0}:=x, \quad x_{n+1}:=R_{T, \gamma_{n}} x_{n} \text { for all } n \in \mathbb{N} .
$$

Then $\left(x_{n}\right) \Delta$-converges to a fixed point of $T$.

We have therefore obtained a new proof of [6, Proposition 1.5]. 


\subsection{Zeros of maximally monotone operators}

In the following, $H$ is a Hilbert space with inner product $\langle\cdot, \cdot\rangle$ and $A: H \rightarrow 2^{H}$ is a maximally monotone operator. We denote by $z \operatorname{er}(A)$ the set of zeros of $A$. Given $\gamma>0$, the resolvent $J_{\gamma A}$ of order $\gamma$ of $A$ is defined by

$$
J_{\gamma A}=\left(i d_{H}+\gamma A\right)^{-1} \text {. }
$$

It is well-known (see, e.g., [9]) that, for every $\gamma>0, J_{\gamma A}: H \rightarrow H$ is a single-valued firmly nonexpansive mapping satisfying $\operatorname{Fix}\left(J_{\gamma A}\right)=z \operatorname{er}(A)$.

Proposition 3.21. Let $\left(\gamma_{n}\right)$ be a sequence of positive real numbers. Then the family $\left(J_{\gamma_{n} A}\right)$ is jointly firmly nonexpansive with respect to $\left(\gamma_{n}\right)$.

Proof. By Proposition 3.14, we can prove, equivalently, that the family $\left(J_{\gamma_{n} A}\right)$ is jointly $\left(P_{2}\right)$ with respect to $\left(\gamma_{n}\right)$. Let $n, m \in \mathbb{N}$ and $x, y \in H$. It is easy to see that

$$
\frac{1}{\gamma_{n}}\left(x-J_{\gamma_{n} A} x\right) \in A\left(J_{\gamma_{n} A} x\right) \text { and } \frac{1}{\gamma_{m}}\left(y-J_{\gamma_{m} A} y\right) \in A\left(J_{\gamma_{m} A} y\right) .
$$

By the monotonicity of $A$ we obtain that

$$
\left\langle J_{\gamma_{n} A} x-J_{\gamma_{m} A} y, \frac{1}{\gamma_{n}}\left(x-J_{\gamma_{n} A} x\right)-\frac{1}{\gamma_{m}}\left(y-J_{\gamma_{m} A} y\right)\right\rangle \geq 0
$$

therefore

$$
\frac{1}{\gamma_{m}}\left\langle J_{\gamma_{n} A} x-J_{\gamma_{m} A} y, y-J_{\gamma_{m} A} y\right\rangle \leq \frac{1}{\gamma_{n}}\left\langle J_{\gamma_{n} A} x-J_{\gamma_{m} A} y, x-J_{\gamma_{n} A} x\right\rangle .
$$

As a consequence of Theorem 3.15 we derive the following well-known weak convergence result (see, e.g., [9, Theorem 23.41.(i)]).

Theorem 3.22. Assume that $z \operatorname{er}(A) \neq \emptyset$ and let $\left(\gamma_{n}\right)$ be a sequence of positive real numbers such that $\sum_{n=0}^{\infty} \gamma_{n}^{2}=\infty$. For any $x \in H$, define the sequence $\left(x_{n}\right)$ by

$$
x_{0}:=x, \quad x_{n+1}:=J_{\gamma_{n} A} x_{n} \text { for all } n \in \mathbb{N} .
$$

Then $\left(x_{n}\right)$ converges weakly to a zero of $A$.

\section{Uniformly firmly nonexpansive and uniformly $\left(P_{2}\right)$ map- pings}

As mentioned in the Introduction, if one wants to obtain strong convergence for the proximal point algorithm, one usually imposes a uniformity condition on the object that is being optimized. The aim of this section is to give such a condition in the abstract setting from the previous section. For a single mapping defined on a Hilbert space, this condition was also considered in [8, Section 3.4], under the name of uniform firm nonexpansivity with a given modulus. We will now generalize this notion to CAT(0) spaces and show how it may be applied for the families of mappings that arise from two of the concrete problems just discussed.

Let $X$ be a $\operatorname{CAT}(0)$ space, $T: X \rightarrow X, C \subseteq X$ be a nonempty subset of $X$ and $\varphi:[0, \infty) \rightarrow$ $[0, \infty)$ be an increasing function which vanishes only at 0 .

Definition 4.1. We say that $T$ is

(i) uniformly firmly nonexpansive on $C$ with modulus $\varphi$ if $T(C) \subseteq C$ and, for all $x, y \in C$ and all $t \in[0,1]$, the following holds:

$$
d^{2}(T x, T y) \leq d^{2}((1-t) x+t T x,(1-t) y+t T y)-2(1-t) \varphi(d(T x, T y)) .
$$


(ii) uniformly $\left(P_{2}\right)$ on $C$ with modulus $\varphi$ if $T(C) \subseteq C$ and, for any $x, y \in C$,

$$
2 d^{2}(T x, T y) \leq d^{2}(x, T y)+d^{2}(y, T x)-d^{2}(x, T x)-d^{2}(y, T y)-2 \varphi(d(T x, T y)) .
$$

Obviously, if $T$ is uniformly firmly nonexpansive (resp. $\left(P_{2}\right)$ ) on $C$, then its restriction $\left.T\right|_{C}$ : $C \rightarrow C$ is firmly nonexpansive (resp. $\left(P_{2}\right)$ ). We remark also that the uniform $\left(P_{2}\right)$ condition may be expressed using the quasi-linearization function as follows:

$$
\langle\overrightarrow{T x T y}, \overrightarrow{y T y}\rangle \leq\langle\overrightarrow{T x T y}, \overrightarrow{x T x}\rangle-\varphi(d(T x, T y)) .
$$

Proposition 4.2. Suppose that $T$ is uniformly firmly nonexpansive on $C$ with modulus $\varphi$. Then $T$ is uniformly $\left(P_{2}\right)$ on $C$ with the same modulus $\varphi$.

Proof. Let $x, y \in C$ and $t \in(0,1)$. As in the proof of Proposition 3.9, we apply the uniform firm nonexpansivity condition and (1) twice to get that

$$
\begin{aligned}
d^{2}(T x, T y) \leq & (1-t)^{2} d^{2}(x, y)+t(1-t) d^{2}(T x, y)+t(1-t) d^{2}(x, T y)+t^{2} d^{2}(T x, T y) \\
& -t(1-t) d^{2}(x, T x)-t(1-t) d^{2}(y, T y)-2(1-t) \varphi(d(T x, T y)) .
\end{aligned}
$$

Divide now by $1-t \neq 0$ to obtain that

$$
\begin{aligned}
(1+t) d^{2}(T x, T y) \leq & (1-t) d^{2}(x, y)+t d^{2}(T x, y)+t d^{2}(x, T y) \\
& -t d^{2}(x, T x)-t d^{2}(y, T y)-2 \varphi(d(T x, T y)),
\end{aligned}
$$

By taking $t \rightarrow 1$ we get what is needed.

As in the non-uniform case, for Hilbert spaces, the two notions coincide.

Proposition 4.3. Assume that $X$ is a Hilbert space and that $T$ is uniformly $\left(P_{2}\right)$ on $C$ with modulus $\varphi$. Then $T$ is uniformly firmly nonexpansive on $C$ with the same modulus $\varphi$.

Proof. Let $x, y \in C$ and $t \in[0,1]$. By the hypothesis, (21) and (4), we immediately get that

$$
\langle T x-T y,(x-T x)-(y-T y)\rangle \geq \varphi(\|T x-T y\|) .
$$

Consequently,

$$
\begin{aligned}
\|((1-t) x+t T x)-((1-t) y+t T y)\|^{2}= & \|(T x-T y)+(1-t)((x-T x)-(y-T y))\|^{2} \\
= & \|T x-T y\|^{2}+(1-t)^{2}\|(x-T x)-(y-T y)\|^{2} \\
& +2(1-t)\langle T x-T y,(x-T x)-(y-T y)\rangle \\
\geq & \|T x-T y\|^{2}+2(1-t) \varphi(\|T x-T y\|) .
\end{aligned}
$$

The following properties will be useful in the proof of our main quantitative result, Theorem 5.1 .

Lemma 4.4. Let $T$ be uniformly $\left(P_{2}\right)$ on $C$ with modulus $\varphi$. Then

$$
\varphi(d(T x, z)) \leq d(x, T x) d(T x, z),
$$

for all $x \in C$ and all $z \in C \cap F i x(T)$.

Proof. Applying (20) for $y:=z$, we get that

$$
d^{2}(T x, z) \leq d^{2}(x, z)-d^{2}(x, T x)-2 \varphi(d(T x, z)) .
$$

It follows that

$$
\begin{aligned}
2 \varphi(d(T x, z)) & \leq d^{2}(x, z)-d^{2}(x, T x)-d^{2}(T x, z) \\
& \leq(d(x, T x)+d(T x, z))^{2}-d^{2}(x, T x)-d^{2}(T x, z) \\
& =2 d(x, T x) d(T x, z) .
\end{aligned}
$$


As an immediate consequence, we obtain

Corollary 4.5. If $T$ is uniformly $\left(P_{2}\right)$ on $C$ with modulus $\varphi$, the set $C \cap F i x(T)$ is at most a singleton.

Proof. Let $x, z \in C \cap \operatorname{Fix}(T)$. Applying Lemma 4.4, we obtain that $\varphi(d(x, z))=0$. Since $\varphi$ vanishes only at 0 , we must have that $x=z$.

We shall now check that the conditions introduced above are indeed satisfied by nontrivial particular cases in the concrete instances that we have presented.

\subsection{Uniformly convex functions}

Let $X$ be a complete $\operatorname{CAT}(0)$ space and $f: X \rightarrow(-\infty, \infty]$ be a proper, convex, lsc function. We use the notation from Subsection 3.2.

Let $\psi:[0, \infty) \rightarrow[0, \infty)$ be an increasing function which vanishes only at 0 and $C \subseteq X$ be nonempty. Recall that $f$ is said to be uniformly convex on $C$ with modulus $\psi$ if for all $x, y \in C$ and all $t \in[0,1]$, the following holds:

$$
f((1-t) x+t y) \leq(1-t) f(x)+t f(y)-t(1-t) \psi(d(x, y)) .
$$

Lemma 4.6. Assume that $f$ is uniformly convex on $C$ with modulus $\psi$. Let $\gamma>0$ be such that $J_{\gamma}(C) \subseteq C$. Then:

(i) for all $u, v \in C$,

$$
d^{2}\left(J_{\gamma} u, v\right) \leq d^{2}(u, v)-d^{2}\left(u, J_{\gamma} u\right)-2 \gamma\left(f\left(J_{\gamma} u\right)-f(v)\right)-2 \gamma \psi\left(d\left(v, J_{\gamma} u\right)\right) .
$$

(ii) for all $x, y \in C$,

$$
d^{2}\left(J_{\gamma} x, J_{\gamma} y\right) \leq d^{2}(x, y)-4 \gamma \psi\left(d\left(J_{\gamma} x, J_{\gamma} y\right)\right) .
$$

Proof. (i) By the definition of $J_{\gamma}$, we have that for all $p \in X$,

$$
f\left(J_{\gamma}(u)\right)+\frac{1}{2 \gamma} d^{2}\left(u, J_{\gamma} u\right) \leq f(p)+\frac{1}{2 \gamma} d^{2}(u, p) .
$$

Let $t \in(0,1)$ be arbitrary. Note that, by $(1)$,

$$
d^{2}\left(u,(1-t) v+t J_{\gamma} u\right) \leq(1-t) d^{2}(u, v)+t d^{2}\left(u, J_{\gamma} u\right)-t(1-t) d^{2}\left(v, J_{\gamma} u\right) .
$$

Applying the first inequality (multiplied by $\gamma$ ) for $p:=(1-t) v+t J_{\gamma} u,(25)$ and the uniform convexity of $f$ on $C$ (since $v, J_{\gamma} u \in C$ ), we get that

$$
\begin{aligned}
\gamma f\left(J_{\gamma} u\right)+\frac{1}{2} d^{2}\left(u, J_{\gamma} u\right) \leq & \gamma\left((1-t) f(v)+t f\left(J_{\gamma} u\right)-t(1-t) \psi\left(d\left(v, J_{\gamma} u\right)\right)\right) \\
& +\frac{1}{2}\left((1-t) d^{2}(u, v)+t d^{2}\left(u, J_{\gamma} u\right)-t(1-t) d^{2}\left(v, J_{\gamma} u\right)\right),
\end{aligned}
$$

hence

$$
\gamma(1-t)\left(f\left(J_{\gamma} u\right)-f(v)\right) \leq \frac{1}{2}(1-t)\left(d^{2}(u, v)-d^{2}\left(u, J_{\gamma} u\right)-t d^{2}\left(v, J_{\gamma} u\right)-2 \gamma t \psi\left(d\left(v, J_{\gamma} u\right)\right)\right) .
$$

Divide by $1-t \neq 0$ and let $t \rightarrow 1$ to obtain that

$$
\gamma\left(f\left(J_{\gamma} u\right)-f(v)\right) \leq \frac{1}{2}\left(d^{2}(u, v)-d^{2}\left(u, J_{\gamma} u\right)-d^{2}\left(v, J_{\gamma} u\right)-2 \gamma \psi\left(d\left(v, J_{\gamma} u\right)\right)\right),
$$

hence (23). 
(ii) Applying (23) with $u:=x, v:=J_{\gamma} y$ and then with $u:=y, v:=J_{\gamma} x$, we get that

$$
\begin{aligned}
& d^{2}\left(J_{\gamma} x, J_{\gamma} y\right) \leq d^{2}\left(x, J_{\gamma} y\right)-d^{2}\left(x, J_{\gamma} x\right)-2 \gamma\left(f\left(J_{\gamma} x\right)-f\left(J_{\gamma} y\right)\right)-2 \gamma \psi\left(d\left(J_{\gamma} x, J_{\gamma} y\right)\right), \\
& d^{2}\left(J_{\gamma} y, J_{\gamma} x\right) \leq d^{2}\left(y, J_{\gamma} x\right)-d^{2}\left(y, J_{\gamma} y\right)-2 \gamma\left(f\left(J_{\gamma} y\right)-f\left(J_{\gamma} x\right)\right)-2 \gamma \psi\left(d\left(J_{\gamma} y, J_{\gamma} x\right)\right) .
\end{aligned}
$$

Summing up, we obtain

$$
2 d^{2}\left(J_{\gamma} x, J_{\gamma} y\right)+d^{2}\left(x, J_{\gamma} x\right)+d^{2}\left(y, J_{\gamma} y\right) \leq d^{2}\left(x, J_{\gamma} y\right)+d^{2}\left(y, J_{\gamma} x\right)-4 \gamma \psi\left(d\left(J_{\gamma} y, J_{\gamma} x\right)\right) .
$$

By (6), we have that

$$
d^{2}\left(x, J_{\gamma} y\right)+d^{2}\left(y, J_{\gamma} x\right) \leq d^{2}(x, y)+d^{2}\left(J_{\gamma} x, J_{\gamma} y\right)+d^{2}\left(x, J_{\gamma} x\right)+d^{2}\left(y, J_{\gamma} y\right),
$$

from where we get our conclusion.

Proposition 4.7. Suppose that $f$ is uniformly convex on $C$ with modulus $\psi$. Let $\gamma>0$ be such that $J_{\gamma}(C) \subseteq C$. Then $J_{\gamma}$ is uniformly firmly nonexpansive on $C$ with modulus $2 \gamma \psi$.

Proof. Let $x, y \in C$ and $t \in[0,1]$. Denote $u:=(1-t) x+t J_{\gamma} x$ and $v:=(1-t) y+t J_{\gamma} y$.

By Proposition 3.16.(iii), we have that $J_{(1-t) \gamma}(u)=J_{\gamma} x$ and $J_{(1-t) \gamma}(v)=J_{\gamma} y$. We get that

$$
\begin{aligned}
d^{2}\left(J_{\gamma} x, J_{\gamma} y\right) & =d^{2}\left(J_{(1-t) \gamma}(u), J_{(1-t) \gamma}(v)\right) \\
& \leq d^{2}(u, v)-4(1-t) \gamma \psi\left(d\left(J_{(1-t) \gamma}(u), J_{(1-t) \gamma}(v)\right) \quad \text { by }(24)\right. \\
& =d^{2}(u, v)-4(1-t) \gamma \psi\left(d\left(J_{\gamma} x, J_{\gamma} y\right) .\right.
\end{aligned}
$$

\subsection{Uniformly monotone operators}

Fix now a Hilbert space $H$ and $C \subseteq H$ a nonempty subset. Let $A: H \rightarrow 2^{H}$ be a multi-valued operator and $\varphi:[0, \infty) \rightarrow[0, \infty)$ be an increasing function which vanishes only at 0 .

Then $A$ is said to be uniformly monotone on $C$ with modulus $\varphi$ (see, e.g. [9, Definition 22.1]) if for all $x, y \in C$ and $u, v \in H$ with $u \in A(x)$ and $v \in A(y)$ we have that

$$
\langle x-y, u-v\rangle \geq \varphi(\|x-y\|) .
$$

Proposition 4.8. Assume that $A$ is a maximally monotone operator which is uniformly monotone on $C$ with modulus $\varphi$. Let $\gamma>0$ be such that $J_{\gamma A}(C) \subseteq C$. Then $J_{\gamma A}$ is uniformly firmly nonexpansive on $C$ with modulus $\gamma \varphi$.

Proof. Let $x, y \in C$. As in the proof of Proposition 3.21, we get that

$$
\left\langle J_{\gamma A} x-J_{\gamma A} y, x-J_{\gamma A} x\right\rangle \geq\left\langle J_{\gamma A} x-J_{\gamma A} y, y-J_{\gamma A} y\right\rangle+\gamma \varphi\left(\left\|J_{\gamma A} x-J_{\gamma A} y\right\|\right) .
$$

Thus, $J_{\gamma A}$ is uniformly $\left(P_{2}\right)$ on $C$ with modulus $\gamma \varphi$. Apply now Proposition 4.3.

\section{A rate of convergence for the uniform case}

We shall now show that in the presence of the uniformity constraint described in the previous section, one indeed gets strong convergence of the proximal point algorithm in its most abstract form, given by Theorem 3.5. Moreover, as announced in the Introduction, we use the tools of proof mining to derive that result from a stronger one which is highly uniform and also quantitative i.e. also yields a rate of convergence for the sequence. 
Let us recall that if $\left(a_{n}\right)_{n \in \mathbb{N}}$ is a convergent sequence in a metric space $(X, d)$ with $\lim _{n \rightarrow \infty} a_{n}=$ $a$, then a rate of convergence of $\left(a_{n}\right)$ is a function $\Phi: \mathbb{N} \rightarrow \mathbb{N}$ such that for all $k \in \mathbb{N}$ and all $n \geq \Phi(k)$,

$$
d\left(a_{n}, a\right) \leq \frac{1}{k+1} .
$$

Another needed quantitative notion will be that of a rate of divergence for a given diverging series $\sum_{n=0}^{\infty} b_{n}=\infty$, which is a function $\theta: \mathbb{N} \rightarrow \mathbb{N}$ such that for all $K \in \mathbb{N}$ we have that $\sum_{n=0}^{\theta(K)} b_{n} \geq K$.

In this section, $X$ is a complete $\operatorname{CAT}(0)$ space and $T_{n}: X \rightarrow X$ for every $n \in \mathbb{N}$. We assume that the family $\left(T_{n}\right)$ has common fixed points and set

$$
F:=\bigcap_{n \in \mathbb{N}} F i x\left(T_{n}\right) \neq \emptyset .
$$

Furthermore, $\varphi:[0, \infty) \rightarrow[0, \infty)$ is an increasing function which vanishes only at 0 and $\left(\gamma_{n}\right)$ is a a sequence in $(0, \infty)$ such that $\sum_{n=0}^{\infty} \gamma_{n}^{2}=\infty$ with rate of divergence $\theta$.

We can state now the main result of this section.

Theorem 5.1. Let $b \in \mathbb{N}, p \in F$ and $C$ be the closed ball of center $p$ and radius $b$. Assume that, for all $n \in \mathbb{N}, T_{n}$ is uniformly $\left(P_{2}\right)$ on $C$ with modulus $\gamma_{n} \varphi$.

For every $x \in C$, let $\left(x_{n}\right)$ be defined by

$$
x_{0}:=x, \quad x_{n+1}:=T_{n} x_{n} \text { for all } n \in \mathbb{N} .
$$

Suppose that $(C 2)$ holds, that is, the sequence $\left(\frac{d\left(x_{n}, x_{n+1}\right)}{\gamma_{n}}\right)$ is nonincreasing.

Then $C \cap F=\{p\}$ and $\left(x_{n}\right)$ converges strongly to $p$ with rate of convergence $\Psi_{b, \theta, \varphi}$, given by

$$
\Psi_{b, \theta, \varphi}(k):=\Sigma_{b, \theta}\left(\left\lceil\frac{2 b}{\varphi\left(\frac{1}{k+1}\right)}\right\rceil\right)+1,
$$

with $\Sigma_{b, \theta}(k):=\theta\left(b^{2}(k+1)^{2}\right)$.

Before proving the theorem, let us give some consequences.

Proposition 5.2. Assume that $H$ is a Hilbert space and $A: H \rightarrow 2^{H}$ is a maximally monotone operator with zer $(A) \neq \emptyset$. Let $b \in \mathbb{N}, p \in z \operatorname{er}(A)$ and $C$ be the closed ball of center $p$ and radius $b$. Suppose that $A$ is uniformly monotone on $C$ with modulus $\varphi$. For any $x \in C$, let $\left(x_{n}\right)$ be defined by (18).

Then $p$ is the unique zero of $A$ in $C$ and $\left(x_{n}\right)$ converges strongly to $p$ with rate of convergence $\Psi_{b, \theta, \varphi}$, given by $(27)$.

Proof. We use the notation from Subsection 3.4. Since, for every $n \in \mathbb{N}, \operatorname{Fix}\left(J_{\gamma_{n} A}\right)=z \operatorname{er}(A) \neq \emptyset$ and $J_{\gamma_{n} A}$ is nonexpansive, it is obvious that $J_{\gamma_{n} A}(C) \subseteq C$. Thus, by Proposition 4.8, every $J_{\gamma_{n} A}$ is uniformly firmly nonexpansive on $C$ with modulus $\gamma_{n} \varphi$. Furthermore, $(C 2)$ is satisfied, by Propositions 3.21 and 3.12. An application of Theorem 5.1 for the family $\left(J_{\gamma_{n} A}\right)$ yields the result.

The above proposition is a quantitative uniform version of Theorem 3.22. If we forget about the quantitative features, we get immediately the following well-known result (see, e.g., [9, Theorem 23.41.(ii)]).

Corollary 5.3. Assume that $H$ is a Hilbert space and $A: H \rightarrow 2^{H}$ is a maximally monotone operator with zer $(A) \neq \emptyset$. Let $\left(\gamma_{n}\right)$ be a sequence of positive real numbers such that $\sum_{n=0}^{\infty} \gamma_{n}^{2}=\infty$, $x \in X$ and $\left(x_{n}\right)$ be defined by (18). Suppose that $A$ is uniformly monotone on every bounded subset of $H$.

Then $\left(x_{n}\right)$ converges strongly to the unique zero of $A$. 
The following result is a quantitative uniform version of Theorem 3.18.

Proposition 5.4. Assume that $X$ is a complete $C A T(0)$ space and $f: X \rightarrow(-\infty, \infty]$ is a convex, proper, lsc function that attains its minimum. Let $b \in \mathbb{N}, p \in \operatorname{Argmin}(f)$ and $C$ be the closed ball of center $p$ and radius $b$. Suppose that $f$ is uniformly convex on $C$ with modulus $\psi$. For any $x \in C$, let $\left(x_{n}\right)$ be defined by (15).

Then $p$ is the unique minimizer of $f$ in $C$ and $\left(x_{n}\right)$ converges strongly to $p$ with rate of convergence $\Omega_{b, \theta, \psi}:=\Psi_{b, \theta, 2 \psi}$.

Proof. By Proposition 3.16, Fix $\left(J_{\gamma_{n}}\right)=\operatorname{Argmin}(f) \neq \emptyset$ and $J_{\gamma_{n}}$ is nonexpansive, hence $J_{\gamma_{n}}(C) \subseteq$ $C$ for all $n$. Use now Proposition 4.7 to get that every $J_{\gamma_{n}}$ is uniformly firmly nonexpansive on $C$ with modulus $2 \gamma_{n} \psi$. Since $(C 2)$ is satisfied (by Propositions 3.17 and 3.12), we can apply Theorem 5.1 for the family $\left(J_{\gamma_{n}}\right)$ to get the result.

\subsection{Proof of Theorem 5.1}

Apply the fact that $C \cap F \neq \emptyset$ and Corollary 4.5 to conclude that $C \cap F=\{p\}$. Since, by Lemma $3.2,\left(x_{n}\right)$ is Fejér monotone with respect to $F$, we have that $d\left(x_{n}, p\right) \leq b$ for all $n \in \mathbb{N}$.

Claim: $\Sigma_{b, \theta}$ is a rate of convergence of the sequence $\left(\frac{d\left(x_{n}, x_{n+1}\right)}{\gamma_{n}}\right)$ towards 0 .

Proof of claim: We reason as in the proof of [34, Lemma 8.3.(ii)]. Let $k \in \mathbb{N}$. By the proof of Lemma 3.3,

$$
\sum_{n=0}^{\infty} d^{2}\left(x_{n}, x_{n+1}\right) \leq b^{2}
$$

Assume that for all $n \in\left\{0, \ldots, \Sigma_{b, \theta}(k)\right\}$ we have that $\frac{d\left(x_{n}, x_{n+1}\right)}{\gamma_{n}}>\frac{1}{k+1}$. It follows that

$$
\sum_{n=0}^{\Sigma_{b, \theta}(k)} d^{2}\left(x_{n}, x_{n+1}\right)>\sum_{n=0}^{\Sigma_{b, \theta}(k)} \gamma_{n}^{2} \frac{1}{(k+1)^{2}}=\frac{1}{(k+1)^{2}} \sum_{k=0}^{\theta\left(b^{2}(k+1)^{2}\right)} \gamma_{n}^{2} \geq b^{2} .
$$

We get a contradiction with (28). Thus, there exists $N \leq \Sigma_{b, \theta}(k)$ such that $\frac{d\left(x_{N}, x_{N+1}\right)}{\gamma_{n}} \leq \frac{1}{k+1}$. By $(C 2)$, the claim follows.

Let $k \in \mathbb{N}$ and $n \geq \Psi_{b, \theta, \varphi}(k)$. Set $n^{\prime}:=n-1$. Then $n^{\prime} \geq \Sigma_{b, \theta}\left(\left\lceil\frac{2 b}{\varphi\left(\frac{1}{k+1}\right)}\right\rceil\right)$, hence, by the claim,

$$
\frac{d\left(x_{n^{\prime}}, x_{n^{\prime}+1}\right)}{\gamma_{n^{\prime}}} \leq \frac{1}{\left\lceil\frac{2 b}{\varphi\left(\frac{1}{k+1}\right)}\right]+1} \leq \frac{1}{\frac{2 b}{\varphi\left(\frac{1}{k+1}\right)}}=\frac{1}{2 b} \cdot \varphi\left(\frac{1}{k+1}\right) .
$$

Applying Lemma 4.4 for $x:=x_{n^{\prime}}, z:=p, T:=T_{n^{\prime}}$ (and hence $\varphi$ becomes $\gamma_{n^{\prime}} \varphi$ ), we get that

$$
\gamma_{n^{\prime}} \varphi\left(d\left(T_{n^{\prime}} x_{n^{\prime}}, p\right)\right) \leq d\left(x_{n^{\prime}}, T_{n^{\prime}} x_{n^{\prime}}\right) d\left(T_{n^{\prime}} x_{n^{\prime}}, p\right) .
$$

Since $x_{n^{\prime}+1}=T_{n^{\prime}} x_{n^{\prime}}$, it follows that

$$
\varphi\left(d\left(x_{n^{\prime}+1}, p\right)\right) \leq \frac{d\left(x_{n^{\prime}}, x_{n^{\prime}+1}\right)}{\gamma_{n^{\prime}}} \cdot d\left(x_{n^{\prime}+1}, p\right) \leq \frac{1}{2 b} \cdot \varphi\left(\frac{1}{k+1}\right) \cdot b=\frac{1}{2} \varphi\left(\frac{1}{k+1}\right) .
$$

If $d\left(x_{n^{\prime}+1}, p\right)>\frac{1}{k+1}$, then $\varphi\left(d\left(x_{n^{\prime}+1}, p\right)\right) \geq \varphi\left(\frac{1}{k+1}\right)>\frac{1}{2} \varphi\left(\frac{1}{k+1}\right)$, since $\varphi$ is increasing and $\varphi\left(\frac{1}{k+1}\right) \neq 0$. We have got a contradiction. Thus, we must have

$$
d\left(x_{n^{\prime}+1}, p\right) \leq \frac{1}{k+1}
$$


which is what we wanted to show, since $n=n^{\prime}+1$.

\section{Acknowledgements:}

Adriana Nicolae was partially supported by DGES (MTM2015-65242-C2-1-P). She would also like to acknowledge the Juan de la Cierva - Incorporación Fellowship Program of the Spanish Ministry of Economy and Competitiveness.

Laurenţiu Leuştean and Andrei Sipoş were partially supported by a grant of the Romanian National Authority for Scientific Research, CNCS - UEFISCDI, project number PN-II-ID-PCE-20113-0383.

\section{References}

[1] D. Ariza-Ruiz, L. Leuştean, G. López-Acedo, Firmly nonexpansive mappings in classes of geodesic spaces, Trans. Amer. Math. Soc. 366, 4299-4322, 2014.

[2] D. Ariza-Ruiz, G. López-Acedo, A. Nicolae, The asymptotic behavior of the composition of firmly nonexpansive mappings, J. Optim. Theory Appl. 167, 409-429, 2015.

[3] M. Bačák, The proximal point algorithm in metric spaces, Israel J. Math. 194, 689-701, 2013.

[4] M. Bačák, Convex analysis and optimization in Hadamard spaces, De Gruyter, 2014.

[5] M. Bačák, U. Kohlenbach, On proximal mappings with Young functions in uniformly convex Banach spaces, J. Convex Anal. 25, 2018.

[6] M. Bačák, S. Reich, The asymptotic behavior of a class of nonlinear semigroups in Hadamard spaces, J. Fixed Point Theory Appl. 16, 189-202, 2014.

[7] M. Bačák, I. Searston, B. Sims, Alternating projections in CAT(0) spaces, J. Math. Anal. Appl. 385, 599-607, 2012.

[8] S. Bartz, H. Bauschke, S. Moffat, X. Wang, The resolvent average of monotone operators: dominant and recessive properties, SIAM J. Optim. 26, 602-634, 2016.

[9] H. Bauschke, P. Combettes, Convex Analysis and Monotone Operator Theory in Hilbert Spaces. Second Edition, Springer, 2017.

[10] I. D. Berg, I. G. Nikolaev, Quasilinearization and curvature of Alexandrov spaces, Geom. Dedicata 133, 195-218, 2008.

[11] H. Bonnel, A. N. Iusem, B. F. Svaiter, Proximal methods in vector optimization, SIAM J. Optim. 15, 953-970, 2005.

[12] H. Brézis, P. L. Lions, Produits infinis de resolvantes, Israel J. Math. 29, 329-345, 1978.

[13] M. R. Bridson, A. Haefliger, Metric spaces of non-positive curvature, Springer, 1999.

[14] E. M. Briseid, Logical aspects of rates of convergence in metric spaces, J. Symbolic Logic 74, $1401-1428,2009$.

[15] F. E. Browder, Convergence of approximants to fixed points of nonexpansive nonlinear mappings in Banach spaces, Arch. Rational Mech. Anal 24, 82-90, 1967.

[16] L. C. Ceng, B. S. Mordukhovich, J. C. Yao, Hybrid approximate proximal method with auxiliary variational inequality for vector optimization, J. Optim. Theory Appl. 146, 267-303, 2010.

[17] S. Dhompongsa, W. A. Kirk, B. Sims, Fixed points of uniformly Lipschitzian mappings, Nonlinear Anal. 65, 762-772, 2006. 
[18] R. Espínola, A. Fernández-León, CAT( $\kappa)$-spaces, weak convergence and fixed points, J. Math. Anal. Appl. 353, 410-427, 2009.

[19] P. Gerhardy, U. Kohlenbach, General logical metatheorems for functional analysis, Trans. Amer. Math. Soc. 360, 2615-2660, 2008.

[20] K. Goebel, S. Reich, Uniform convexity, hyperbolic geometry, and nonexpansive mappings, Marcel Dekker, 1984.

[21] O. Güler, On the convergence of the proximal point algorithm for convex minimization, SIAM J. Control Optim. 29, 403-419, 1991.

[22] B. Halpern, Fixed points of nonexpanding maps, Bull. Amer. Math. Soc. 73, 957-961, 1967.

[23] A. N. Iusem, T. Pennanen, B. F. Svaiter, Inexact variants of the proximal point algorithm without monotonicity, SIAM J. Optim 13, 1080-1097, 2003.

[24] J. Jost, Equilibrium maps between metric spaces, Calc. Var. Partial Diff. Equations 2, 173204, 1994.

[25] J. Jost, Convex functionals and generalized harmonic maps into spaces of non positive curvature, Comment. Math. Helvetici 70, 659-673, 1995.

[26] A. Kaplan, R. Tichatschke, Proximal point methods and nonconvex optimization, J. Global Optim. 13, 389-406, 1998.

[27] W. A. Kirk, Geodesic geometry and fixed point theory, Seminar of Mathematical Analysis, Colecc. Abierta, 64, Univ. Sevilla Secr. Publ., 195-225, 2003.

[28] W. A. Kirk, B. Panyanak, A concept of convergence in geodesic spaces, Nonlinear Anal. 68, $3689-3696,2008$.

[29] U. Kohlenbach, Theorie der majorisierbaren und stetigen Funktionale und ihre Anwendung bei der Extraktion von Schranken aus inkonstruktiven Beweisen: Effektive Eindeutigkeitsmodule bei besten Approximationen aus ineffektiven Beweisen, PhD Thesis, Goethe University Frankfurt, 1990.

[30] U. Kohlenbach, Some logical metatheorems with applications in functional analysis, Trans. Amer. Math. Soc. 357, 89-128, 2005.

[31] U. Kohlenbach, Applied proof theory: Proof interpretations and their use in mathematics, Springer Monographs in Mathematics, Springer, 2008.

[32] U. Kohlenbach, Recent progress in proof mining in nonlinear analysis, IFCoLog Journal of Logics and their Applications 10, 3357-3406, 2017.

[33] U. Kohlenbach, Proof-theoretic methods in nonlinear analysis, draft, 2017; to appear in Proceedings of the ICM2018.

[34] U. Kohlenbach, L. Leuştean, A. Nicolae, Quantitative results on Fejér monotone sequences, Commun. Contemp. Math. 20, 1750015, 42 pp., 2018.

[35] U. Kohlenbach, G. López-Acedo, A. Nicolae, Quantitative asymptotic regularity for the composition of two mappings, Optimization 66, 1291-1299, 2017.

[36] U. Kohlenbach, G. López-Acedo, A. Nicolae, Moduli of regularity and rates of convergence for Fejér monotone sequences, arXiv:1711.02130 [math.OC], 2017.

[37] U. Kohlenbach, P. Oliva, Proof mining: a systematic way of analysing proofs in mathematics, Proc. Steklov Inst. Math. 242, 136-164, 2003. 
[38] A. Koutsoukou-Argyraki, Effective rates of convergence for the resolvents of accretive operators, Numer. Funct. Anal. Optim. 38, 1601-1613, 2017.

[39] T. Kuczumow, An almost convergence and its applications, Ann. Univ. Mariae CurieSklodowska Sect. A 32, 79-88, 1980.

[40] L. Leuştean, Nonexpansive iterations in uniformly convex $W$-hyperbolic spaces, in: A. Leizarowitz, B.S. Mordukhovich, I. Shafrir, A. Zaslavski (eds.), Nonlinear Analysis and Optimization I: Nonlinear Analysis, Cont. Math. 513, Amer. Math. Soc., 193-209, 2010.

[41] L. Leuştean, A. Sipoş, An application of proof mining to the proximal point algorithm in CAT(0) spaces, in: A. Bellow, C. Calude, T. Zamfirescu (eds.), Mathematics Almost Everywhere. In Memory of Solomon Marcus, World Scientific, 2018.

[42] L. Leuştean, A. Sipoş, Effective strong convergence of the proximal point algorithm in CAT(0) spaces, arXiv:1801.02179 [math.OC], 2018; to appear in Journal of Nonlinear and Variational Analysis.

[43] T. C. Lim, Remarks on some fixed point theorems, Proc. Amer. Math. Soc. 60, 179-182, 1976.

[44] B. Martinet, Régularisation d'inéquations variationnelles par approximations successives, Rev. Française Informat. Recherche Opérationnelle 4, 154-158, 1970.

[45] U. F. Mayer, Gradient flows on nonpositively curved metric spaces and harmonic maps, Comm. Anal. Geom. 6, 199-253, 1998.

[46] A. Papadopoulos, Metric spaces, convexity and nonpositive curvature, IRMA Lectures in Mathematics and Theoretical Physics 6, European Math. Soc., 2005.

[47] R. T. Rockafellar, Monotone operators and the proximal point algorithm, SIAM J. Control Optim. 14, 877-898, 1976. 